\title{
Immediate Type Hypersensitivity to Heparins: Two Case Reports and a Review of the Literature
}

\author{
Philipp Cesana $^{a}$ Kathrin Scherer ${ }^{b}$ Andreas J. Bircher ${ }^{b}$ \\ ${ }^{\mathrm{a}}$ Department of Dermatology, and ${ }^{\mathrm{b}}$ Allergology Unit, University Hospital Basel, Basel, Switzerland
}

\section{Established Facts}

- Heparins are widely used anticoagulants in clinical practice, and they are known to provoke all types of hypersensitivity reactions; especially delayed type hypersensitivity reactions (type IV) are common.

- Immediate type hypersensitivity reactions to heparin compounds are very rare, and only a few cases have been published so far.

\section{Novel Insights}

- In immediate type hypersensitivity reactions the causative agent can be identified with a skin test, which suggests an IgE-mediated reaction.

- Therapeutic alternatives with a small risk of cross-reactivity in case of a sensitization to a low-molecular-weight heparin are unfractioned heparin and especially the pentasaccharid fondaparinux.

- Heparins of the same substance class, such as low-molecular-weight heparins, should be avoided as alternative substances.

\section{Keywords}

Drug allergy · Immediate type hypersensitivity · Heparin ·

Fondaparinux $\cdot$ Cross-reactivity

\section{Abstract \\ Immediate type hypersensitivity reactions due to heparins are rare, and the exact immunologic pathomechanism has not been identified so far. In our 2 case reports, we describe first a 50-year-old female who received dalteparin (Frag- $\min ^{\circledR}$ ) and developed signs of an immediate type hypersen- sitivity reaction. The personal history revealed a previous ap-}

plication of dalteparin (Fragmin ${ }^{\circledR}$ ). Evaluation with a skin prick test showed positive results for dalteparin. The second case deals with a 73-year-old female with a suspected immediate type reaction after the administration of dalteparin (Fragmin ${ }^{\circledR}$ ). A skin prick test was negative but intracutaneous tests showed a positive reaction to the causative agent. Both cases indicated cross-reactivity reactions for low-molecular-weight heparin (LMWH) but not for unfractioned heparin (UFH) or fondaparinux. In conclusion, our case reports including a review of published cases of immediate type hypersensitivity reactions after the application of heparins illustrate this rare complication. Mostly, the causative

\section{KARGER}

(c) 2017 S. Karger AG, Basel

E-Mail karger@karger.com

www.karger.com/iaa
Correspondence to: Prof. Dr. Andreas J. Bircher

Department of Dermatology, University Hospital Basel

Petersgraben 4

$\mathrm{CH}-4031$ Basel (Switzerland)

E-Mail andreas.bircher@unibas.ch 
agent can be identified with a skin test, which is highly suggestive of an IgE-mediated reaction. Therapeutic alternatives for patients with sensitization to an LMWH are UFH and fondaparinux. Both agents have a small risk of cross-reactivity compared to heparins of the same substance class.

(c) 2017 S. Karger AG, Basel

\section{Introduction}

Heparins are widely used therapeutic agents for prophylaxis and treatment of thromboembolic diseases [1]. Chemically, heparins are composed of a complex mixture of polysaccharide chains and, based on their diverse lengths and molecular weights, unfractionated heparin (UFH), low-molecular-weight heparin (LMWH), and ultra-LMWH (ULMWH) can be distinguished. These heparins are the most commonly used in clinical practice. Other anticoagulants like heparinoids (danaparoid) or direct thrombin inhibitors (lepirudin) are used generally in patients with heparin-induced thrombopenia (HIT) because of their small therapeutic index [2].

Heparins are known to provoke all types of hypersensitivity reactions such as type I, type II, type III, and type IV reactions according to the classification by Gell and Coombs. However, immediate type hypersensitivity reactions (type I) are very rare and only a few documented cases have been published so far [3]. A recent epidemic of anaphylactic reactions to heparins could be related to oversulfated heparin molecules resulting in activation of the contact system [4].

\section{Case Report 1}

A 50-year-old female presented at the emergency department with a high suspicion of deep venous thrombosis. A few minutes after receiving a subcutaneous injection of dalteparin $\left(\right.$ Fragmin $\left.^{\circledR}\right)$, she immediately developed pruritic wheals on her whole body, a swollen tongue and swollen eyelids, dyspnea, and finally a short loss of consciousness. After the administration of antihistamines and corticosteroids, she recovered quickly. Her personal history revealed that she had been anticoagulated prophylactically with dalteparin $\left(\right.$ Fragmin $\left.^{\circledR}\right)$ some months ago after vein stripping. At that time, no allergic reactions were documented.

After this episode, she was diagnosed with venous thrombosis of the soleus vein and was anticoagulated with the direct factor Xa inhibitor rivaroxabaran (Xarelto ${ }^{\circledR}$ ) without further problems, and she was referred to the Department of Allergology for additional testing within 4 months.

With the initial hypothesis of an immediate type hypersensitivity reaction after the application of dalteparin (Fragmin ${ }^{\circledR}$ ), the following anticoagulants were tested: unfractioned sodium heparin
(Liquemin $\left.{ }^{\circledR}\right)$, LMWH sodium dalteparin $\left(\right.$ Fragmin $\left.{ }^{\circledR}\right)$, calcium nadroparin $\left(\right.$ Fraxiparin $\left.{ }^{\circledR}\right)$, and sodium enoxaparin $\left(\right.$ Clexane $\left.^{\circledR}\right)$, as well as the ULMWH fondaparinux $\left(\operatorname{Arixtra}^{\circledR}\right)$. A skin prick test was positive for dalteparin only, and secondary symptoms like wheals, beginning angioedema of the lips, and dyspnea were observed. After oral administration of levocetirizin $\left(\mathrm{Xyzal}^{\circledR}\right)$ and recurrent salbutamol inhalations (Ventolin ${ }^{\circledR}$ ), she rapidly recovered. Skin prick tests with all other heparins were negative. Intracutaneous tests were weakly positive at a concentration of 1:100 and clearly positive at a dilution of 1:10 for LMWH such as nadroparin and enoxaparin. With unfractioned heparin (UFH) and the pentasaccharid fondaparinux, respectively, the intracutaneous tests remained negative. A basophil activation test (BAT) showed no activation for dalteparin, enoxaparin, and fondaparinux (Table 1).

Therefore, the patient was diagnosed with a specific type I hypersensitivity to all tested LMWH and no sensitization to UFH or the ULMWH fondaparinux. An intravenous challenge test with UFH (cumulative dose of 7,500 IE Liquemin ${ }^{\circledR}$ ) and subcutaneous provocation with ULMWH fondaparinux (cumulative dose of 2.5 mg Arixtra ${ }^{\circledR}$ ) were tolerated without any symptoms.

\section{Case Report 2}

A 73-year-old female patient was treated 5 years ago with dalteparin $\left(\right.$ Fragmin ${ }^{\circledR}$ ) because of a lung embolism. After the application of dalteparin, the patient reported pruritus with local swelling of the hands; however, an allergologic work-up was not performed at that time. Because of a planned operation of the paranasal sinuses, the question of a tolerated anticoagulant was raised.

With an initial hypothesis of an immediate type hypersensitivity reaction of grade I (angioedema of the hands) after the application of dalteparin $\left(\right.$ Fragmin $\left.^{\circledR}\right)$, a skin prick test with the same reagents as described in case 1 was performed. All tested reagents were negative. In intracutaneous tests, the only positive reaction at a dilution of 1:100 was found for the LMWH nadroparin. All other LMWH, as well as UFH and the pentasaccharid fondaparinux, werenegative. In the BAT dalteparin, nadroparin, and fondaparinux did not show significant activation or degranulation of the basophil granulocytes (Table 2).

With a documented specific type I hypersensitivity to the LMWH nadroparin, a subcutaneous challenge test with the structurally least related ULMWH fondaparinux was performed (cumulative dose of $2.5 \mathrm{mg}$ Arixtra ${ }^{\circledR}$ ) and it was tolerated without any reaction.

\section{Discussion}

Heparins may provoke type I to type IV hypersensitivity reactions; particularly delayed type hypersensitivity (DTH) reactions (type IV) are common [3]. After a latency of 2-10 days to 3 weeks, infiltrating eczematous plaques can develop locally at the injection sites. Histological findings are lympho-histiocytic dermal infiltration and spongiosis of the epidermis. Only rarely generalized maculopapular exanthemas and exceptionally Lyell syndrome have been described $[3,5]$. The most danger- 
Table 1. Case 1: results of the diagnostic tests

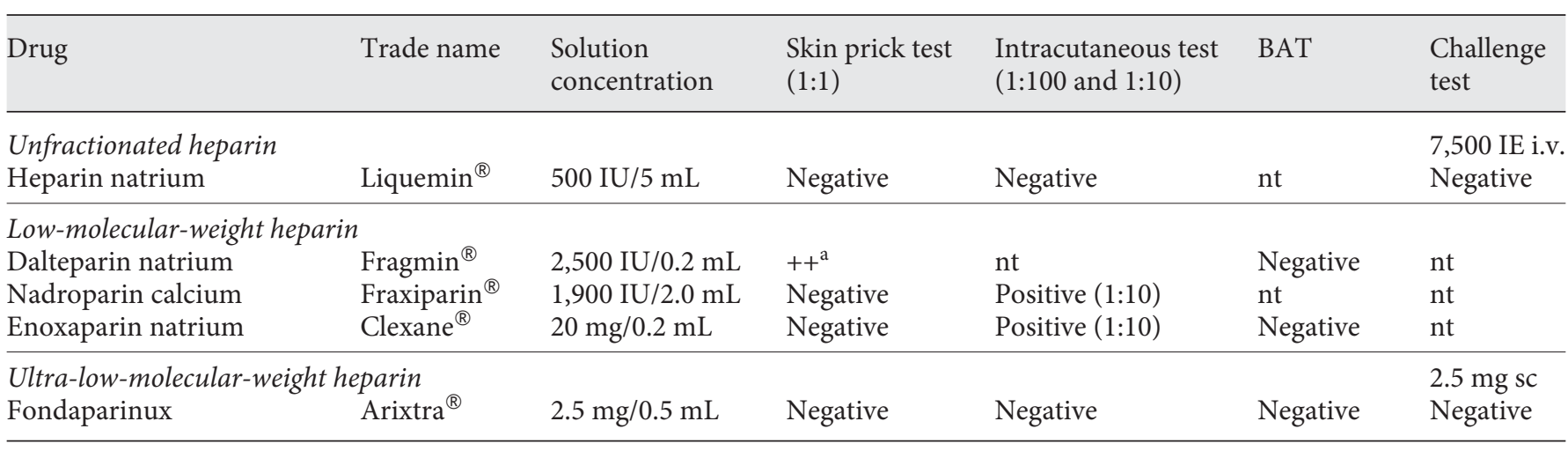

Skin tests were read after 20 min. nt, not tested; i.v., intravenous; sc, subcutaneously; BAT, basophil activation test. ${ }^{a}$ Wheals, beginning angioedema of the lips, and dyspnea.

Table 2. Case 2: Results of the diagnostic tests

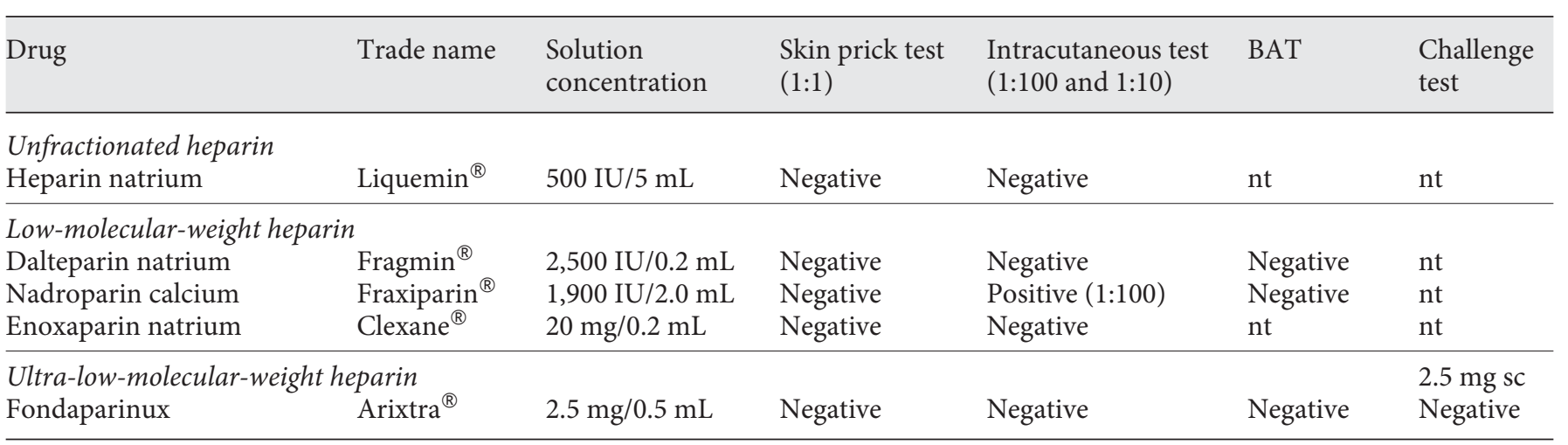

Skin tests were read after 20 min.nt, not tested; sc, subcutaneously; BAT, basophil activation test.

ous hypersensitivity reaction to systemic heparin use is heparin-induced thrombocytopenia (HIT II), a type II antibody-mediated hypersensitivity reaction against complexes of platelet factor 4 and heparin [2].

Immediate type hypersensitivity reactions to heparin compounds seem to be very rare, and only a few cases have been published so far [6-18]. Based on skin tests, the most likely pathomechanism seems to involve an IgEmediated reaction. Nevertheless, a complete allergologic work-up was not performed on all reported patients and only 10 cases have been completely followed up so far. An allergologic work-up is crucial to detecting the responsible agent and to identifying alternative substances to treat patients. Due to the low prevalence of immediate type hypersensitivity reactions to heparins, the most reliable diagnostic tool is uncertain. Standard allergological testing for immediate type hypersensitivity usually includes in vivo tests (skin prick and intradermal tests and a challenge test), as well as in vitro tests (sIgE and BAT). Skin tests have a high sensitivity for DTH reactions from heparins, and especially intracutaneous testing and subcutaneous reexposure can be considered gold standards for identification of the suspected elicitor [19].

In Table 3, all published cases of immediate type hypersensitivity reactions to heparins with a complete allergologic work-up are listed $[10-16,18,20]$. Analogous to our 2 cases, in most patients the heparin causative of the immediate type hypersensitivity reaction was an LMWH. In $83.3 \%$ of cases, the substance causative of the immediate type hypersensitivity reaction could be detected by skin tests (skin prick test/intracutaneous skin test), and in the other cases at least some positive skin tests results for the same substance class have been found. Therefore, as in DTH reactions, skin test seems to have a high sensitiv- 
Table 3. Results of allergologic work-ups in published cases with immediate type hypersensitivity to heparins

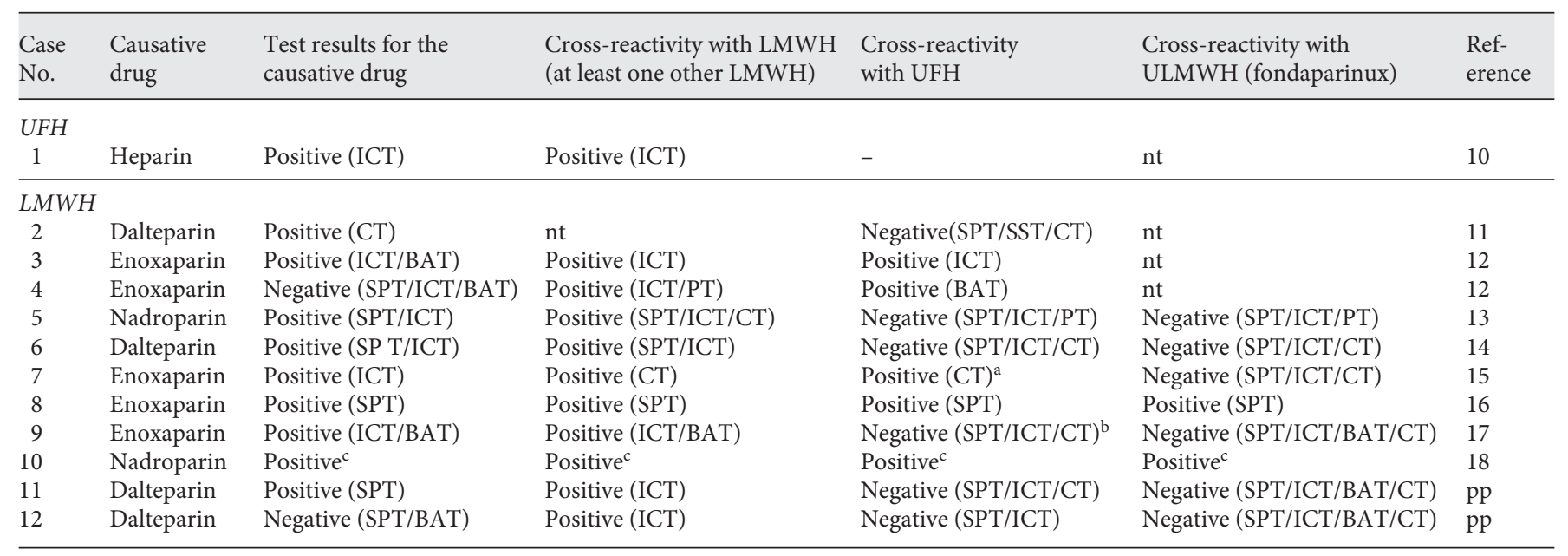

UFH, unfractioned heparin; LMWH, low-molecular-weight heparin; ULMWH, ultra-low-molecular-weight heparin; SPT, skin prick test; SST, skin scratch test; ICT, intracutaneous test; PT, patch test; BAT, basophil activation test; CT, challenge test; nt, not tested; pp, present publication. ${ }^{a}$ Successful desensitization afterwards. ${ }^{\mathrm{b}}$ BAT was (false) positive. ${ }^{\mathrm{c}}$ Positive results with SPT, ICT, and BAT: not otherwise specified.

ity for detection of the causative drug in immediate type hypersensitivity reactions.

The diagnostic significance of the BAT is still unclear. Cabarello et al. [12] postulated that the BAT is a useful, safe, and reliable test for in vitro diagnosis of a heparin allergy, which permits avoidance of risky challenge tests. However, the BAT seems to have false-positive and falsenegative reactions [20], and therefore its diagnostic significance and reliability remain controversial. In our 2 cases the BAT was negative for the suspicious elicitors.

Cross-reactions among heparin preparations in DTH are a known issue and detection of an alternative substance is often cumbersome [21]. Evidence regarding cross-reactions in immediate type hypersensitivity is lacking. All published cases so far, including our 2 reported cases (Table 3), have shown cross-reactivity to at least a second LMWH. Therefore, alternative LMWH are not considered safe for patients with a known sensitization to LWMH. Cross-reactions with UFH have been reported in $45.5 \%$ of cases. However, Kavut and Koca [15] showed that desensitization with UFH was found to be a safe option in immediate type hypersensitivity to LMWH.

Interestingly, the factor $\mathrm{Xa}$ inhibitor fondaparinux showed only a low incidence of cross-reactivity (25\%) and may be a good alternative for patients with sensitization to LMWH. In 5 out of 8 tested cases, the compatibility of fondaparinux as an alternative anticoagulant was proven with a subcutaneous challenge test. In 1 case all skin tests were negative and a provocation test was not performed
[13]. The other 2 cases where fondaparinux was not considered a safe alternative anticoagulant involved a positive skin prick test [15] and a not-otherwise-specified crossreaction to fondaparinux [17]. However, no clinical immediate type hypersensitivity reaction to fondaparinux has been published so far, and the allergic potential for the development of a DTH reaction is known to be significantly lower compared to other heparins (0.4 vs. 7.5\%). Furthermore, fondaparinux-induced thrombocytopenia (HIT) is a rarity [5,22]. This might be due to the full synthetic structure of the pentasaccharide sequence of heparin with a molecular weight of only $1,728 \mathrm{kDa}$ [23].

In DTH reactions, Weberschock et al. [21] showed in a recent review that in patients with a known sensitization to heparins the risk of a cross-reaction to other heparins was very high (92.9\%). Furthermore, they reported a probability of $67.1 \%$ for the development of a cross-reactivity reaction to UFH. The lowest frequency of crossreactions was observed for pentosan polysulfate (36.4\%) and fondaparinux (10.4\%). Fondaparinux is therefore recommended as the current best alternative also in cases of DTH reactions to either UFH or LMWH. These conclusions are comparable to our results and indicate that, independently of a DTH or an immediate type hypersensitivity reaction, the risk for a cross-reaction to another specific heparin molecule seems to be similar, even if the pathomechanisms are different. Nevertheless the exact antigenic moieties and the mechanisms involved have not been identified so far $[24,25]$. 
New anticoagulants in oral applicable forms, such as the direct thrombin inhibitor dabigatran $\left(\operatorname{Pradaxa}^{\circledR}\right)$ or the direct factor Xa inhibitors rivaroxaban (Xarelto ${ }^{\circledR}$ ) and apixaban (Eliquis ${ }^{\circledR}$ ), were admitted in recent years for several indications. Due to their structural differences they may be additional alternatives for patients with a heparin allergy [26].

\section{Conclusions}

Immediate type hypersensitivity reactions to heparins are very rare and only a few cases have been published so far. Among all of the cases, only one third of the patients have had further allergologic testing. According to the published data and our 2 cases, the causative agent can be identified in most cases with a skin test, and these results suggest an IgE-mediated reaction.

Therapeutic alternatives in case of sensitization to an LMWH are UFH, with an average risk, and the structurally least related pentasaccharid fondaparinux, which has the lowest risk for cross-reactivity. Heparins of the same substance class such as LMWH should be avoided as alternative substances. However, all alternatives should always be identified by a challenge test.

\section{Disclosure Statement}

The authors have no conflict of interests to declare.

\section{References}

1 Gray E, Mulloy B, Barrowcliffe TW: Heparin and low-molecular-weight heparin. Thromb Haemost 2008;99:807-818.

2 Arepally GM, Ortel TL: Clinical practice: heparin-induced thrombocytopenia. N Engl J Med 2006;355:809-817.

3 Bircher AJ, Harr T, Hohenstein L, Tsakiris DA: Hypersensitivity reactions to anticoagulant drugs: diagnosis and management options. Allergy 2006;61:1432-1440.

4 Kishimoto TK, Viswanathan K, Ganguly T, Elankumaran S, Smith S, Pelzer K, Lansing JC, Sriranganathan N, Zhao G, GalchevaGargova Z, et al: Contaminated heparin associated with adverse clinical events and activation of the contact system. N Engl J Med 2008;358:2457-2467.

5 Schindewolf M, Lindhoff-Last E, Ludwig RJ, Boehncke WH: Heparin-induced skin lesions. Lancet 2012;380:1867-1879.

6 Bottio T, Pittarello G, Bonato R, Fagiolo U, Gerosa G: Life-threatening anaphylactic shock caused by porcine heparin intravenous infusion during mitral valve repair. J Thorac Cardiovasc Surg 2003;126:1194-1195.

7 Tiu A, Pang JM, Martin R, Officer N: Allergic reactions to enoxaparin and heparin: a case report and review of the literature. $\mathrm{N} Z$ Z Med J 2004;117:U1126.

8 Basic-Jukic N, Kes P: Severe anaphylactic reaction in a haemodialysis patient after administration of reviparin. Nephrol Dial Transplant 2004;19:1021-1022.

9 Parissis H: Lepirudin as an alternative to "heparin allergy" during cardiopulmonary bypass. J Cardiothorac Surg 2011;6:44.

10 Berkun Y, Haviv YS, Schwartz LB, Shalit M: Heparin-induced recurrent anaphylaxis. Clin Exp Allergy 2004;34:1916-1918.
11 Ueda A, Nagase S, Morito N, Yotsumoto M, Ohba S, Hasegawa Y, Narita M, Koyama A: Anaphylactoid reaction induced by low-molecular-weight heparin in a hemodialysis patient. Nephron 2001;87:93-94.

12 Caballero MR, Fernandez-Benitez M: Allergy to heparin: a new in vitro diagnostic technique. Allergol Immunopathol (Madr) 2003; 31:324-328.

13 Bekkenk MW, van Zuuren EJ: Immediate type hypersensitivity after injection of nadroparin (Fraxiparin). Thromb Haemost 2005; 94:673-674.

14 Harr T, Scherer K, Tsakiris DA, Bircher AJ: Immediate type hypersensitivity to low molecular weight heparins and tolerance of unfractioned heparin and fondaparinux. Allergy 2006;61:787-788.

15 Kavut AB, Koca E: Successful desensitization with un-fractionated heparin in a patient with heparin allergy and tolerance to fondaparinux. Asian Pac J Allergy Immunol 2012;30:162166.

16 Anders D, Trautmann A: Allergic anaphylaxis due to subcutaneously injected heparin. Allergy Asthma Clin Immunol 2013;9:1.

17 Gelardi M, Ciprandi G, Buttafava S, Quaranta $\mathrm{N}$, Squeo V, Incorvaia C, Frati F: Nasal inflammation in Parietaria-allergic patients is associated with pollen exposure. J Investig Allergol Clin Immunol 2014;24:352-353.

18 Balthazar S, Watremez C, Vigneault L, Eeckhoudt S, Pirson F, Hermans C: Management of anticoagulation during cardiopulmonary bypass in a patient with allergy to heparin and heparin-like compounds: a case-report. Blood Coagul Fibrinolysis 2014;25:900-903.
19 Pfohler C, Muller CS, Pindur G, Eichler H, Schafers HJ, Grundmann U, Tilgen W: Delayed-type heparin allergy: diagnostic procedures and treatment alternatives - a case series including 15 patients. World Allergy Organ J 2008;1:194-199.

20 Gonzalez P, de la Sen ML, Venegas I, Ramon A, Soriano V, Cueva B, Fernandez J: Immediate hypersensitivity to heparins: a cross-reactivity study. J Investig Allergol Clin Immunol 2014;24:367-368.

21 Weberschock T, Meister AC, Bohrt K, Schmitt J, Boehncke WH, Ludwig RJ: The risk for cross-reactions after a cutaneous delayedtype hypersensitivity reaction to heparin preparations is independent of their molecular weight: a systematic review. Contact Dermatitis 2011;65:187-194.

22 Maetzke J, Hinrichs R, Staib G, ScharffetterKochanek K: Fondaparinux as a novel therapeutic alternative in a patient with heparin allergy. Allergy 2004;59:237-238.

23 Petitou M, Herault JP, Bernat A, Driguez PA, Duchaussoy P, Lormeau JC, Herbert JM: Synthesis of thrombin-inhibiting heparin mimetics without side effects. Nature 1999;398:417422.

24 Gaigl Z, Pfeuffer P, Raith P, Brocker EB, Trautmann A: Tolerance to intravenous heparin in patients with delayed-type hypersensitivity to heparins: a prospective study. $\mathrm{Br} \mathrm{J}$ Haematol 2005;128:389-392.

25 Jappe U: Allergy to heparins and anticoagulants with a similar pharmacological profile: an update. Blood Coagul Fibrinolysis 2006; 17: 605-613.

26 Scherer Hofmeier K, Bircher AJ: Hypersensitivity reactions to modern antiplatelet and anticoagulant drugs. Allergo J Int 2015;24:5866.
Immediate Type Hypersensitivity to Heparins
Int Arch Allergy Immunol 2016;171:285-289 DOI: $10.1159 / 000453525$ 